\title{
Glucocorticoid repression of human with-no-lysine (K) kinase-4 gene expression is mediated by the negative response elements in the promoter
}

\author{
Chunyi $\mathrm{Li}^{1 *}$, Yan $\mathrm{Li}^{1 *}$, Yinghui $\mathrm{Li}^{1}$, Hong Liu ${ }^{1}$, Zhijun Sun ${ }^{1,2}$, Jingyu $\mathrm{Lu}^{1}$ and Yanyan Zhao ${ }^{1}$ \\ ${ }^{1}$ Department of Medical Genetics, China Medical University, No. 92, Bei Er Road, Shenyang, Liaoning 110001, China \\ ${ }^{2}$ Department of Cardiovascular Medicine, The Second Affiliated Hospital, China Medical University, Shenyang, Liaoning 110004, China \\ (Correspondence should be addressed to Y Zhao; Email: yyzhao@mail.cmu.edu.cn)
}

*(C Li and Y Li contributed equally to this work)

\begin{abstract}
With-no-lysine (K) kinase-4 (WNK4) is a serine/threonine kinase that plays an essential role in the regulation of fluid and electrolyte homeostasis. The effects of glucocorticoids, key physiological regulators, on the WNK4 gene expression are still unknown. Here, we used dexamethasone (Dex) to treat the human embryo kidney 293 (HEK293) cells and found a decrease of human WNK4 ( $h W N K 4$ ) mRNA level by northern blot and real-time quantitative PCR. After an $h W N K 4$ transcriptional initiation site was located by $5^{\prime}$ rapid amplification of cDNA end assay, a series of $5^{\prime}$-deleted $h W N K 4$ promoter-luciferase constructs were generated by PCR. Transfection of these constructs in COS-7 and HEK293 cells revealed that Dex inhibited the $h W N K 4$ transcriptional activity in glucocorticoid receptor (GR)-dependent pattern. Two negative glucocorticoid response elements (nGREs) were identified at -285 and -337 of the $h W N K 4$ gene promoter and the GR binding activity to them was increased by Dex as shown by electrophoretic mobility shift assay and chromatin immunoprecipitation. In summary, these data demonstrated that $h W N K 4$ was a new glucocorticoid-regulated gene whose expression was inhibited through the interaction of GR with nGREs in the promoter region.
\end{abstract}

Journal of Molecular Endocrinology (2008) 40, 3-12

\section{Introduction}

Glucocorticoids are secreted by the adrenal glands and play an important role in metabolism, maintenance of homeostasis, cell proliferation, and differentiation (Buckingham 2006). As medication, glucocorticoids are widely prescribed for anti-inflammatory and immunosuppressive therapy, administered to pregnant individuals to accelerate fetal pulmonary development, and used as a component in many chemotherapy regimens for some cancers. However, long-term use of glucocorticoids has been limited by the adverse effects, including endocrine, metabolic, musculoskeletal, gastrointestinal, dermatological, and fluid and electrolyte perturbations, in addition to the development of glucocorticoid resistance. These physiological and pharmacological effects as well as the undesired side effects of glucocorticoids are hypothesized due to the regulation of gene transcription in different types of cells.

It is well known that glucocorticoids increase water, sodium, and chloride reabsorption as well as potassium and calcium excretion both inside and outside of the kidney to modulate fluid and electrolyte balance. For example, prolonged glucocorticoid administration often leads to electrolyte disturbance and hypertension, and the prenatal administration increases the possibilities of sodium retention and hypertension in later life (Ortiz et al. 2003, Dickinson et al. 2007). In order to demonstrate the molecular basis of glucocorticoid-mediated fluid and electrolyte balance, it is necessary to find the effects of glucocoricoids on target molecules involved in electrolyte homeostasis in epithelia.

With-no-lysine (K) kinase-4 (WNK4) is a novel serine/threonine kinase lacking a characteristic lysine in the ATP-docking site and plays an important role in regulation of ion transport process. Human WNK4 ( $h W N K 4)$ gene, when mutated, causes pseudohypoaldosteronism type II (OMIM no. 145260) featuring hypertension and hyperkalemia due to excessive renal sodium chloride and potassium retention (Wilson et al. 2001). The discovery of mutations in the $h W N K 4$ gene has generated considerable interests in its function and the molecular mechanism leading to electrolyte disturbance and hypertension. Recent studies have shown that WNK4 is expressed in multiple chloride-transporting epithelia and predominantly expressed in the distal convoluted tubule, connecting tubule, and collecting duct. It is subcellularly localized near tight junctions, along lateral membranes, and within the cytoplasm. Experimental 
observations demonstrated that WNK4 is a natural inhibitor of diverse epithelial transporters. It inhibits the $\mathrm{NaCl}$ cotransporter and the renal outer medullary potassium channel activities via a kinase-independent mechanism, and increases paracellular chloride flux by phosphorylation of multiple claudins (Kahle et al. 2003, 2004, Yamauchi et al. 2004). Based on its anatomical and functional characters, WNK4 is proposed to regulate transcellular and paracellular ion flux across diverse epithelia and act as a molecular switch for electrolyte homeostasis. As mentioned above, most of the studies focused on the function of WNK4 gene, while little is known about its upstream regulator. In this current study, we aim to identify whether $W N K 4$ is one of the targets for glucocorticoid-mediated electrolyte balance by investigating the effects of glucocorticoids on the $h W N K 4$ expression and the mechanisms.

\section{Materials and methods}

\section{Cell culture and RNA isolation}

Human embryo kidney 293 (HEK293) and African green monkey kidney (COS-7) cells were maintained in Dulbecco's Modified Eagle's Medium (GIBCO) with $10 \%$ fetal bovine serum, $100 \mathrm{unit} / \mathrm{ml}$ penicillin, and $100 \mu \mathrm{g} / \mathrm{ml}$ streptomycin at $37^{\circ} \mathrm{C}$ in a humidified atmosphere containing $5 \% \mathrm{CO}_{2}$. To examine the effect of dexamethasone (Dex; Sigma-Aldrich) on the $h W N K 4$ gene expression, HEK293 cultures were switched to serum-free media and then exposed to $1 \mu \mathrm{M}$ Dex or $1 \mu \mathrm{M}$ Dex with $10 \mu \mathrm{M}$ RU486 (SigmaAldrich) for $24 \mathrm{~h}$. Total RNA was extracted from these cells using TRIzol reagent (Invitrogen).

\section{Northern blotting analysis}

Total RNA $(20 \mu \mathrm{g})$ was fractionated in a $1.2 \%$ agaroseformaldehyde gel and then transferred onto a nylon membrane Hybond-N (Amersham Biosciences). A 321 bp antisense cRNA from $h W N K 4$ cDNA was labeled, hybridized, and detected by CDP-Star using DIG Northern starter kit (Roche) according to the manufacturer's instructions. Signals of hybridization bands were detected on X-ray film and quantified by densitometric analyses. After exposure to film (5-60 min), the membrane was stripped and then rehybridized with the $\beta$-actin probe for normalization.

\section{Reverse transcription and real-time quantitative PCR}

Total RNA $(1 \mu \mathrm{g})$ was reversely transcribed with random primers using reverse transcription reagent kit (Promega) following the manufacturer's protocol. Real-time quantitative PCR was performed in an ABI Prism 7500
Sequence Detection System (Applied Biosystems, Foster City, CA, USA) using a FAM-labeled probe, 5'-FAMCTTCCTGCTCCCGCCGCCGC-Eclipse- ${ }^{\prime}$, and a pair of primers specific to the $h W N K 4$ transcript giving a $144 \mathrm{bp}$ product, forward 5'-GTGAAGGCTGCGGAAGACTC-3' and reverse $5^{\prime}$-CTGGGTCTCCATGTCCTCCTT- ${ }^{\prime}$. The $25 \mu$ reaction mixtures contained $10 \mathrm{mM}$ Tris- $\mathrm{HCl}$ (pH 8.3), $50 \mathrm{mM} \mathrm{KCl}, 1.5 \mathrm{mM} \mathrm{MgCl}_{2}, 200 \mu \mathrm{M}$ dNTPs, $1.25 \mathrm{U}$ Taq polymerase, $40 \mathrm{ng}$ template cDNA, and $150 \mathrm{nM}$ probe and primers (400 $\mathrm{nM}$ for each). All PCR reagents were from TaKaRa Biotech (Dalian, China). The reaction condition was $95^{\circ} \mathrm{C}$ for 3 min followed by 40 cycles at $95^{\circ} \mathrm{C}$ for $15 \mathrm{~s}$ and $60^{\circ} \mathrm{C}$ for $50 \mathrm{~s}$. As an internal control, $\beta$-actin was measured under the same conditions in real-time quantitative PCR with a FAMlabeled probe, $5^{\prime}$ FAM-CCAGGGCGTGATGGTGGGCAT-Eclipse $3^{\prime}$, and a pair of primers specific for $\beta$-actin transcript giving a $155 \mathrm{bp}$ product, forward 5'-CCGT CTTCCCCTCCATCG-3' ${ }^{\prime}$ and reverse $5^{\prime}$-GTCCCAGTT GGTGACGATGC-3'. Cycle threshold values $\left(C_{\mathrm{t}}\right)$ were analyzed by the SDS1.4 software (Applied Biosystems) and relative quantification of $h W N K 4$ expression was determined using the comparative $C_{\mathrm{t}}$ method (ABI Prism 7500, SDS User Bulletin; Applied Biosystems). Each sample was amplified in triplicate to obtain average $C_{\mathrm{t}}$ value. Reaction without cDNA templates was used as negative control. Results were expressed as relative $h W N K 4$ expression compared with the untreated control that was taken as $100 \%$.

\section{$5^{\prime}$ rapid amplification of CDNA end (5'-RACE) assay}

Human Kidney Marathon-Ready cDNA amplification kit (Clontech) was used according to the supplier's instructions. Forward primers were a 27-mer adaptor primer (AP1) and a 23-mer nested AP2. Reverse primers were gene-specific primers (GSP) of the $h W N K 4$ gene exon 1 including outer primer (GSP1) and inner primer (GSP2): 5'-ACGCGCGGAGTCTTCCGCAGCCT- ${ }^{\prime}$ and $5^{\prime}$-CTGGGACATGAGGACGGTGGTCT- ${ }^{\prime}$. The firstround PCR was performed using template MarathonReady cDNA and primers AP1 and GSP1. PCR product was diluted for the second-round PCR using primers AP2 and GSP2. The PCR products were analyzed by agarose gel electrophoresis. DNA from major band was cloned into the pMD18-T vector (TaKaRa Biotech) and then sequenced.

\section{Plasmid construction}

The luciferase reporter plasmids used in this study were derived from pGL3-Basic (Promega) lacking eukaryotic promoter and enhancer. The PCR fragment comprising -484 to +62 of the $h W N K 4$ gene was generated using human genomic DNA with forward primer 
5'-CACTGACGTCTCCGTTCGGC-3' ${ }^{\prime}$ and reverse primer $5^{\prime}$-GACATGAGGACGGTGGTCTC-3'. This PCR product was cloned into pMD18-T vector and subcloned into the unique $K p n I / H i n d I I I$ sites of pGL3-Basic vector to generate a reporter construct p484-luc. A series of $5^{\prime}$-deleted constructs were derived from the p484-luc by PCR. The forward primers were 5'-CGCACAAACAGGTT$3^{\prime}$ (-337), $5^{\prime}$-TTTGCTCACTCTTAGTGCGG-3' (-325), $5^{\prime}$-CACTCAGTTCTGG-3' (-285), $5^{\prime}$-TGGCCTCAGAGTGAGACT- $3^{\prime} \quad(-275), \quad 5^{\prime}$-GAAAGAAGGGGACGC-3' $(-241), 5^{\prime}$-GGCGGTGACTAAGGTGAG-3' (-216), and $5^{\prime}$-AGCGAGTCCGTCTGTCAG-3' (-52). The reverse primer was the same one as used in the construction of the p484-luc. All constructs were confirmed by sequencing with no coding frame shift in the luciferase gene. For transient transfection, the plasmids were prepared using Mid Pre plasmid kits (Qiagen).

\section{Transient transfection and luciferase assays}

HEK293 or COS-7 cells were seeded in 24-well plates, grown to $80-90 \%$ confluence, and transfected by Lipofectamine 2000 (Invitrogen) according to the manufacturer's protocol with $1 \mu \mathrm{g}$ reporter construct, with or without $0.5 \mu \mathrm{g}$ expression vector coding for human glucocorticoid receptor (GR; pRShGR $\alpha$ ). Cotransfection of $0 \cdot 01 \mu \mathrm{g}$ pRL-TK (Promega), a plasmid encoding for Renillaluciferase, was performed to normalize transfection efficiencies. After $24 \mathrm{~h}$, cells were treated with Dex $(1 \mu \mathrm{M})$ in the presence or absence of RU486 $(10 \mu \mathrm{M})$ and incubated for $24 \mathrm{~h}$. Cells were then harvested and luciferase activities were measured using the Dual-Luciferase Reporter Assay system (Promega) on Lumat LB9507 luminometer (Bethold Technologies, Bad Wildbad, Germany).

\section{Nuclear extracts preparation and electrophoretic mobility shift assay (EMSA)}

Nuclear extracts from HEK293 cells with or without Dex treatment were prepared and EMSA was carried out as previously described (Zhao et al. 1999). Briefly, oligonucleotides corresponding to the putative glucocorticoid response motifs at the $h W N K 4$ promoter

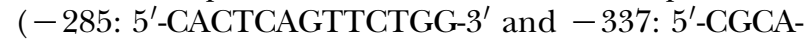
CAAACAGGTT- $\left.3^{\prime}\right)$ and mutant oligonucleotides with underlined mutated bases (5'-CACTTAATTATGG- $3^{\prime}$ and $5^{\prime}$-CGCAATAACAAGTT-3' ${ }^{\prime}$ ) were synthesized and annealed. glucocorticoid response element (GRE) consensus oligonucleotides were purchased from Promega and anti-GR antibody was purchased from Santa Cruz Biotechnology (Santa Cruz, CA, USA). Probes were end radiolabeled with $\left[\gamma^{32} \mathrm{P}\right]$ ATP (Furui Biotechnology, Beijing, China) using T4 polynucleotide kinase (TaKaRa Biotech). Binding reactions were performed in a volume of $30 \mu \mathrm{l}$ containing $\sim 5000 \mathrm{cpm}$ labeled probe, $12 \mu \mathrm{g}$ nuclear extracts, $1 \mu \mathrm{g} / \mu \mathrm{l}$ poly $(\mathrm{dI}-\mathrm{dC})$, and $1 \times$ binding buffer $(10 \mathrm{mM}$ HEPES, $50 \mathrm{mM} \mathrm{KCl}, 5 \mathrm{mM} \mathrm{MgCl} 2,0.5 \mathrm{mM}$ EDTA, $1 \mathrm{mM}$ dithiothreitol) on ice for $30 \mathrm{~min}$. For competition assays, 100- or 500-fold molar excess of the unlabeled double-stranded DNA was added to the reaction mixture. For supershift assays, the anti-GR antibody was incubated for $30 \mathrm{~min}$ at room temperature before the probe was added. Reaction mixtures were loaded onto an $8 \%$ polyacrylamide gel and run at $150 \mathrm{~V}$ in $0 \cdot 375 \times$ tris-borate-EDTA (TBE) for $4 \mathrm{~h}$. The gel was dried under vacuum and autoradiographed.

\section{Chromatin immunoprecipitation (ChIP) assay}

HEK293 cells were treated with or without Dex for $24 \mathrm{~h}$. Formaldehyde $(1 \%)$ was added to the medium and incubated for $20 \mathrm{~min}$ at $37^{\circ} \mathrm{C}$. Cells were harvested, resuspended in lysis buffer (1\% SDS, $10 \mathrm{mM}$ EDTA, $50 \mathrm{mM}$ Tris- $\mathrm{HCl}(\mathrm{pH} 8 \cdot 1)$ ), incubated at $4{ }^{\circ} \mathrm{C}$ for $10 \mathrm{~min}$, and then sonicated to generate approximate 100-300 bp DNA fragments. One-third of the lysate was used as DNA input control. The remaining twothirds were diluted tenfold with ChIP dilution buffer $(0.01 \%$ SDS, $1 \%$ Triton X-100, $1 \mathrm{mM}$ EDTA, $10 \mathrm{mM}$ Tris-HCl, $150 \mathrm{mM} \mathrm{NaCl}$ ) followed by incubation with specific anti-GR antibody or with nonimmune rabbit immuno-globulin $\mathrm{G}(\mathrm{IgG})$ as a negative control at $4{ }^{\circ} \mathrm{C}$ overnight. Immunoprecipitated complexes were collected by protein A/G-agarose beads, washed, and incubated at room temperature for $20 \mathrm{~min}$. Crosslinking of protein-DNA complexes was reversed at $65^{\circ} \mathrm{C}$ for $5 \mathrm{~h}$. Then, DNA was extracted with phenol/chloroform. PCR was carried out in $25 \mu \mathrm{l}$ for 30 cycles using the primers specific for the $h W N K 4$ promoter. The set of primers was as follows: forward $5^{\prime}$-CACTGACGTCTCCGTTCGGC-3' $(-484)$ and reverse $5^{\prime}$-CGAGCAGCCCCTCACCTTAG-3' $(-189)$ for a $296 \mathrm{bp}$ fragment, forward 5'-CACTGACCTCTCCGTTCGGC-3' $(-484)$ and reverse $5^{\prime}$-ggagcatcctccegcactaa-3' $3^{\prime}(-295)$ for a $190 \mathrm{bp}$ fragment, and forward $5^{\prime}$-TCCCACTCAGTTCTGGCCTCA-3' $(-288)$ and reverse 5'-CGAGCAGCCCCTCACCTTAG-3' ( -189$)$ for a $100 \mathrm{bp}$ fragment. PCRs using neuronal nitric oxide synthase (nNOS) gene-specific promoter primers (forward $5^{\prime}$-ATGTGGAAGACAGCATAGACC- $3^{\prime}$ and reverse $5^{\prime}$-AGAGGCAAAGAGGAAAACCACCA- $3^{\prime}$ ) to generate a $189 \mathrm{bp}$ fragment without GR binding were used as a negative control. All PCR signals stained with ethidium bromide in $1.5 \%$ agarose gels were quantified by densitometric analyses to calculate the amount of PCR products. The values were normalized to that of input and shown as a percentage of untreated controls, which were taken as $100 \%$ for each group. All in vivo ChIP assays were 
repeated at least thrice with similar results and a representative result was shown.

\section{Densitometric analyses and statistical analysis}

All densitometric analyses were performed using LabWorks Image Acquisition and Analysis Software (Media Cybernetics, UVP Inc., Cambridge, UK). Data were shown as mean \pm s.E.M. from at least three independent experiments. Differences between quantitative datasets were analyzed by one-way ANOVA or $t$-test as appropriate and $P<0.05$ was considered significant.

\section{Results}

\section{Suppression of the $h W N K 4$ mRNA by glucocorticoids in HEK293 cells}

To assess whether glucocorticoids are implicated in modulation of the $h W N K 4$ expression, we initially detected the $h W N K 4$ mRNA level in HEK293 cells
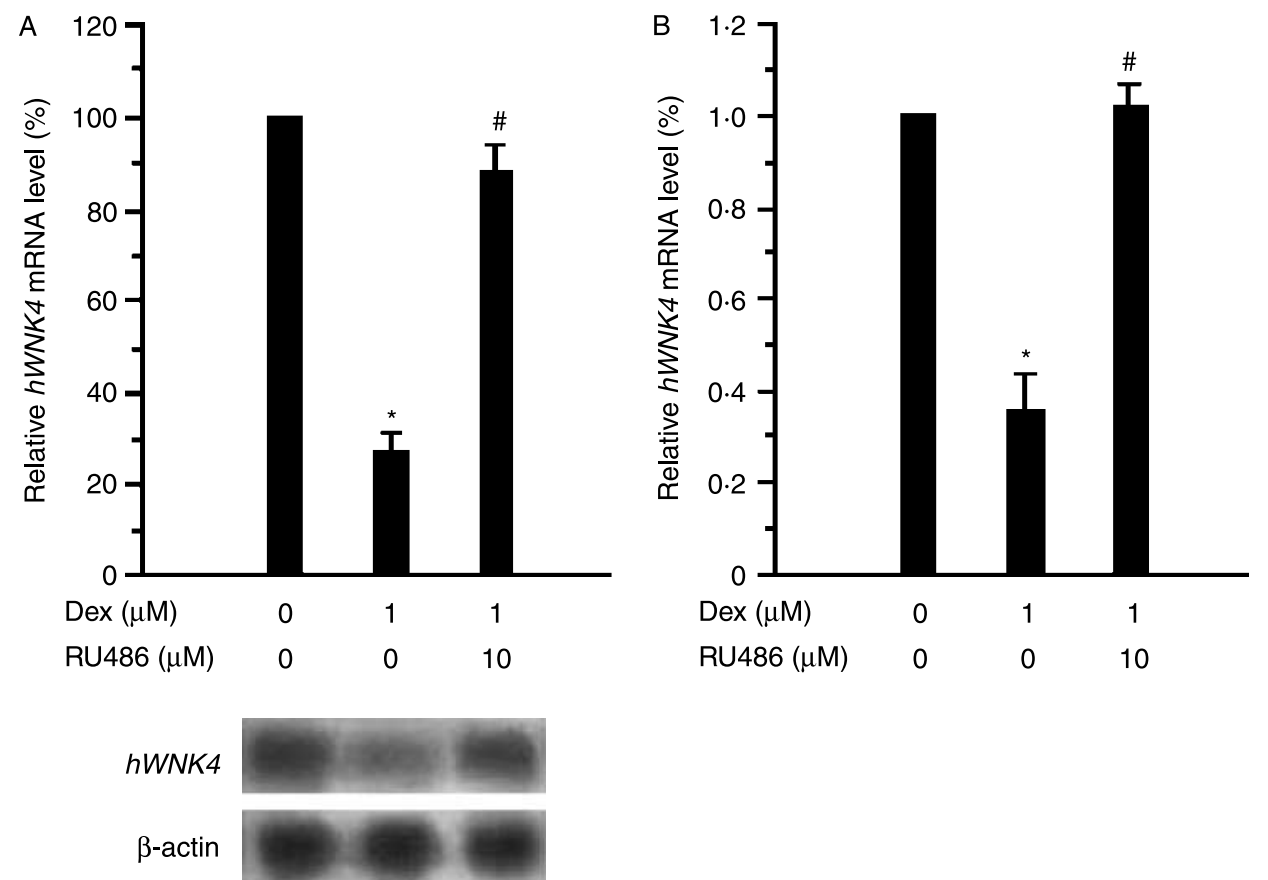

Figure 1 Effect of Dex on $h W N K 4$ mRNA expression level in HEK293 cells. HEK293 cells were exposed to $1 \mu \mathrm{M}$ Dex with or without $10 \mu \mathrm{M}$ RU486 for $24 \mathrm{~h}$. (A) Northern blot analysis was used to determine the expression of $h W N K 4$ mRNA. The $h W N K 4$ mRNA levels were quantitated by densitometric analysis, normalized to $\beta$-actin. Results are expressed as a percentage of untreated control that is taken as $100 \%$. The bar is mean \pm S.E.M. from three independent experiments $\left({ }^{\star} P<0.01\right.$ compared with untreated control; ${ }^{*} P<0.01$ compared with Dex alone). Representative northern blot of $h W N K 4$ and $\beta$-actin are shown in the lower panel. (B) Real-time PCR was used to determine the expression of $h W N K 4$ mRNA using the comparative $C_{\mathrm{t}}$ method. $\beta$-actin was used for normalization. Results are expressed as a percentage of untreated control that is taken as $100 \%$. The bar is mean \pm S.E.M. from three independent experiments. $\left({ }^{*} P<0.01\right.$ compared with untreated control; ${ }^{*} P<0.01$ compared with Dex alone). 


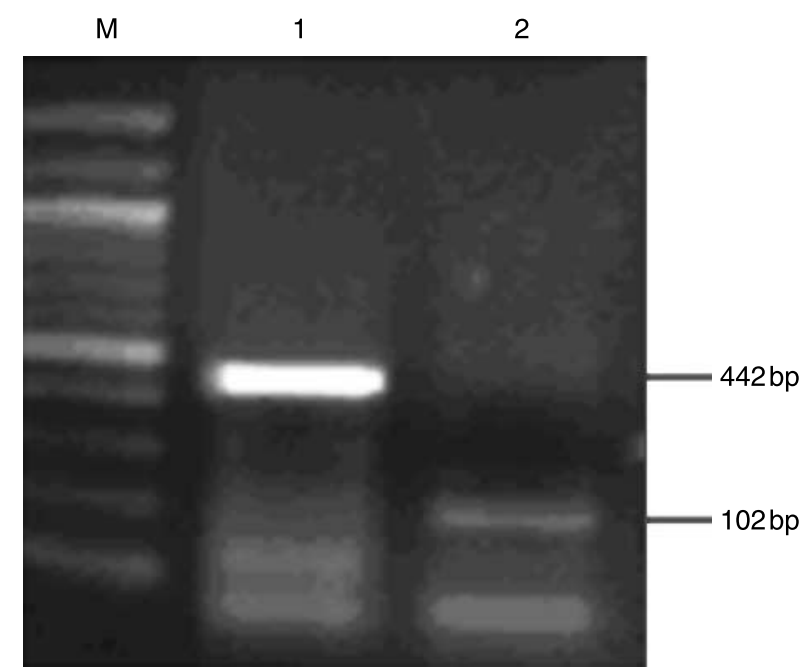

Figure 2 Determination of transcription initiation site of the hWNK4 gene by $5^{\prime}$-RACE. A product of the first-round PCR was the $442 \mathrm{bp}$ band and nested PCR product was the $102 \mathrm{bp}$ band. $\mathrm{M}, 100$ bp DNA ladder.

5'-flanking sequence (GenBank AC016889) was analyzed for transcription factor binding sites with Match (public version 1.0) search (Kel et al. 2003). Results showed that the promoter exhibited no TATA-box motif, while a putative initiator element, which was thought to be strongly required for the transcription initiator activity of TATA-less promoter, was found at position -1 (CTACCCT). It conformed well to a consensus initiator sequence PyPyANA/TPyPy except for the underlined $\mathrm{C}$. We also found several potential transcription factor binding sites in this region, such as GR, Sp1, AP-2 $\alpha$, and C/EBP (Fig. 3).

\section{Repression of $h W N K 4$ promoter activity through GR}

To confirm the suppression of $h W N K 4$ mRNA level by glucocorticoids through GR, we transfected p484-luc (an $h W N K 4$ promoter luciferase reporter construct spanning -484 to +62 ) with or without GR expression vector in COS-7 cells that do not contain endogenous GR (Oakley et al. 1999) and therefore provide a null background for GR expression. Luciferase assay showed that in the context of GR, Dex treatment reached the maximal suppression of the promoter activity (Fig. 4A). Furthermore, Dex-suppressed $h W N K 4$ promoter activity in a dose-dependent pattern (Fig. 4B). These results suggested that the suppression of the $h W N K 4$ promoter activity by glucocorticoids was in a GR-dependent pattern and the $484 \mathrm{bp}$ upstream sequence of the $h W N K 4$ gene was sufficient to confer the suppression of $h W N K 4$ gene transcription by glucocorticoids.

\section{Characteristics of the basal activity of the $h W N K 4$ promoter}

To delineate potential transcriptional regulatory elements, a series of luciferase reporter constructs containing $5^{\prime}$-flanking deletions of the $h W N K 4$ promoter

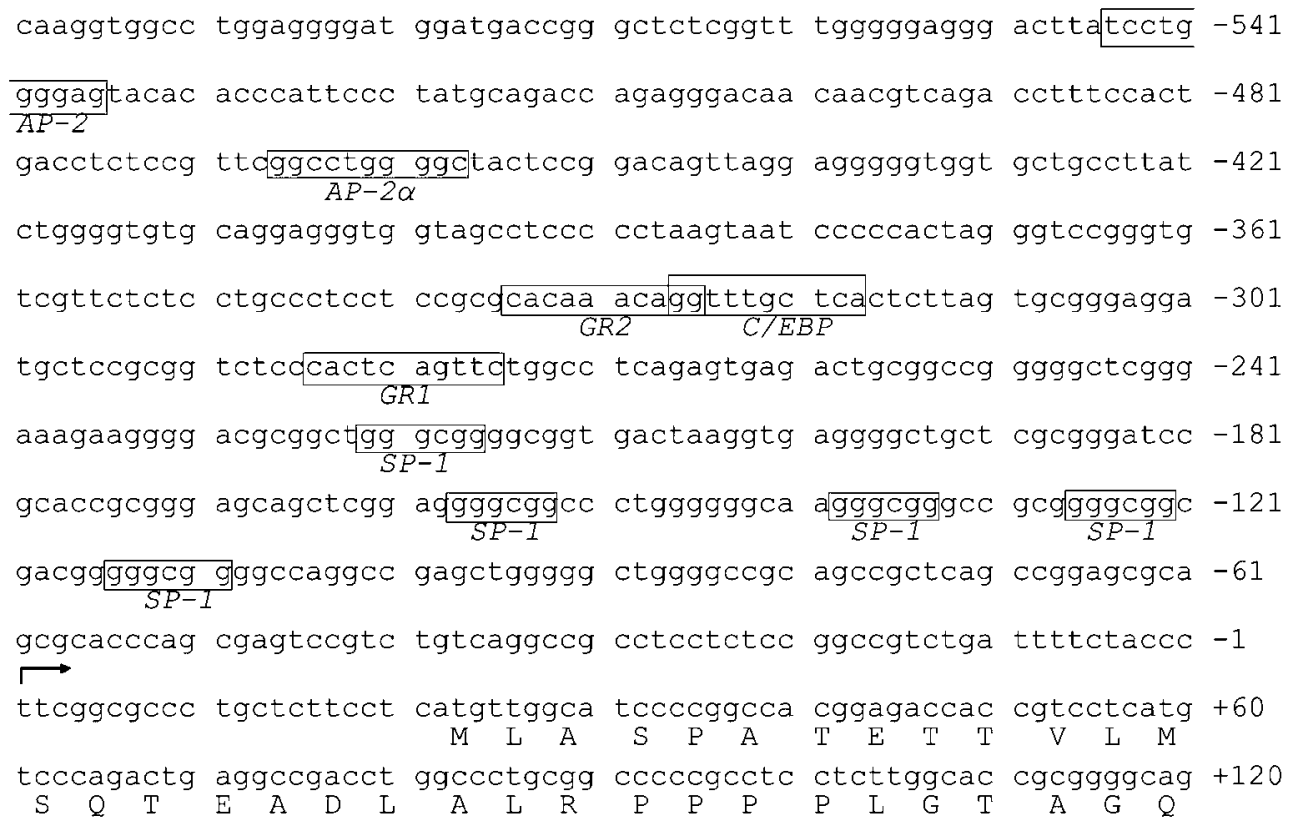

Figure 3 Nucleotide sequence of the $5^{\prime}$-flanking region of $h W N K 4$ gene (GenBank AC016889). Potential transcription factor binding motifs are boxed including the two glucocorticoid receptor elements (GR1 and GR2). The transcription initiation site is indicated by a bent arrow. 

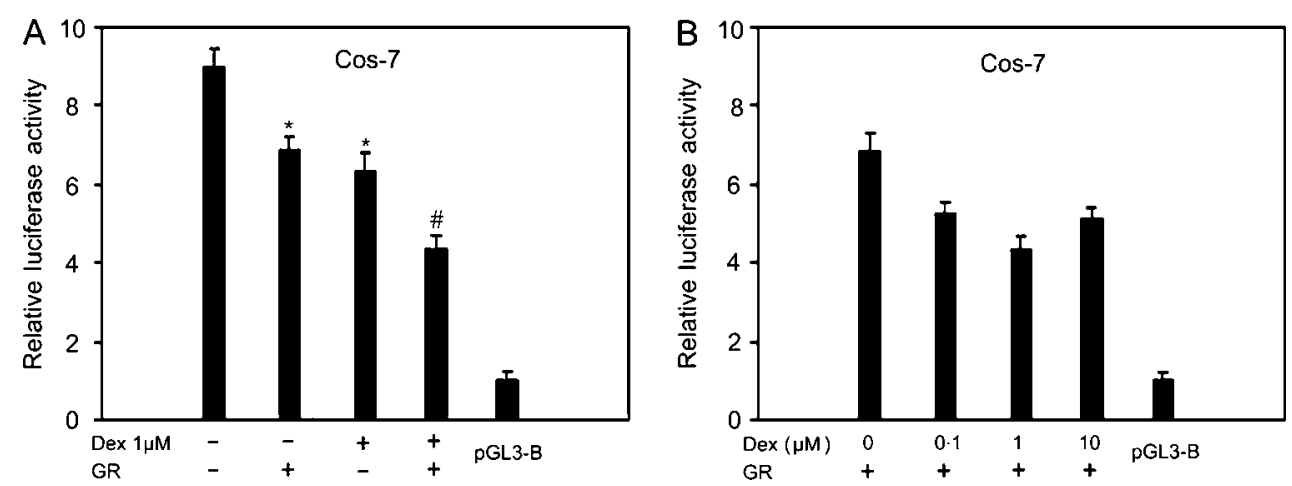

Figure 4 Promoter activity of p484-luc (an $h W N K 4$ promoter luciferase reporter construct spanning -484 to +62 ) in COS-7 cells. (A) The p484-luc construct was cotransfected with $(+)$ or without $(-)$ GR vector into COS-7 cells in absence $(-)$ or presence $(+)$ of $1 \mu \mathrm{M}$ Dex. (B) COS-7 cells were cotransfected with p484-luc construct and GR vector and treated with Dex at the indicated concentrations for $24 \mathrm{~h}$. Luciferase activities were measured by a luminometer (Bethold), normalized to Renilla luciferase activities. Results are expressed as the fold of pGL3-Basic vector that is taken as 1 . The bar is mean \pm S.E.M. from three independent experiments in duplicate for each construct $\left({ }^{\star} P<0.05\right.$ compared with control; ${ }^{\#} P<0.01$ compared with control).

were generated (Fig. 5A) and transiently transfected into HEK293 cells. Luciferase assay of the basal promoter activity showed that there were multiple cis-acting elements essential for the promoter activity. A region between -216 and -52 was necessary for optimal activity. The deletion of p337-luc construct to -325 and p285-luc construct to -275 resulted in the increased promoter activity, implying that $12 \mathrm{bp}$ from -337 to -325 and $10 \mathrm{bp}$ from -285 to -275 were negative regulatory elements. In addition, three regions -484 to $-337,-325$ to -285 , and -275 to -52 played positive regulatory role in the transcription of $h W N K 4$ gene (Fig. 5B).

\section{Glucocorticoid-related elements in the $h W N K 4$ promoter}

Our attention is focused on the association of the negative elements with the Dex-reduced promoter activity. Significant decrease of promoter activity was observed in p484-luc, p337-luc, and p285-luc constructs, but not in p325-luc and p275-luc. Furthermore, the Dexreduced activity was effectively abolished by RU 486, a GR antagonist, confirming that these effects required GR binding (Fig. 5C). These observations suggested that negative GREs (nGREs) were located at positions -285 (nGRE1) and - 337 (nGRE2) in the $h W N K 4$ promoter.

\section{Identification of two functional nGREs in the $h W N K 4$ promoter}

To identify whether GR binding to the putative binding sites $(-285$ and -337$)$, we synthesized nGRE1 and nGRE2 oligonucleotides and performed EMSA with HEK293 nuclear extracts. Both nGRE1 and nGRE2 probes formed a strong complex with nuclear extracts that were competed by 100 - or 500 -fold molar excess of unlabeled nGRE1 and nGRE2 respectively (Fig. 6A), but not by 500-fold excess of unlabeled nGRE1 and nGRE2 mutant (in Fig. 6B and C). The GRE consensus binding was also competed by 100 -fold molar excess of unlabeled nGRE1 or nGRE2 probe (Fig. 6A). Further studies showed that their binding activities were blocked by anti-GR antibody, but not by IgG control (Fig. 6B and C) and enhanced with Dex-treated nuclear extracts (Fig. 6D and E).

To confirm the results of EMSA, we performed in vivo ChIP assay. After chromatin from Dex-treated and -untreated cells was immunoprecipitated with anti-GR antibody, we amplified -484 to -189 region containing both nGRE1 and nGRE2, -484 to -295 region containing nGRE2, and -288 to -189 region containing nGRE1 and obtained 296, 190, and $100 \mathrm{bp}$ products respectively (Fig. 7A). All bindings were specific, as both immunoprecipitation with nonimmune IgG and with an anti-p65 antibody (data not shown) did not show $h W N K 4$ promoter fragments. Moreover, when nuclear extracts were immunoprecipitated with the anti-GR antibody, no fragment was detected by PCRs using nNOS-specific primers, which was used to identify a nuclear-factor kappaB (NF-kB) binding site previously (Li et al. 2007; Fig. 7B). The amount of PCR products can reflect the level of GR binding in ChIP. Therefore, we quantified the PCR products and found that the amounts of 296, 190, and $100 \mathrm{bp}$ products in Dex-treated group were higher compared with those in the Dex-untreated group (Fig. 7C). These indicated that nGRE1 and nGRE2 were located at position -285 and -337 of the $h W N K 4$ promoter and Dex treatment resulted in a significant increase of GR binding to both sites. 


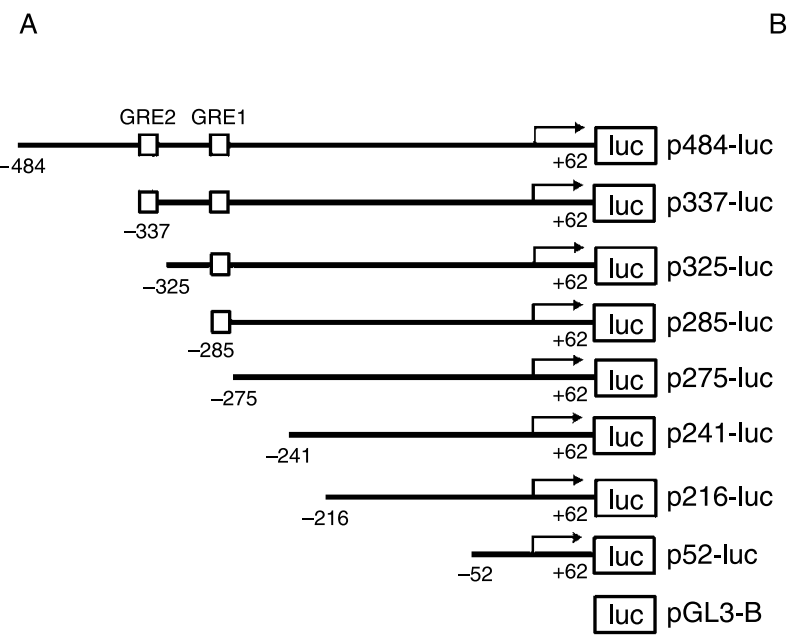

B Relative luciferase activity
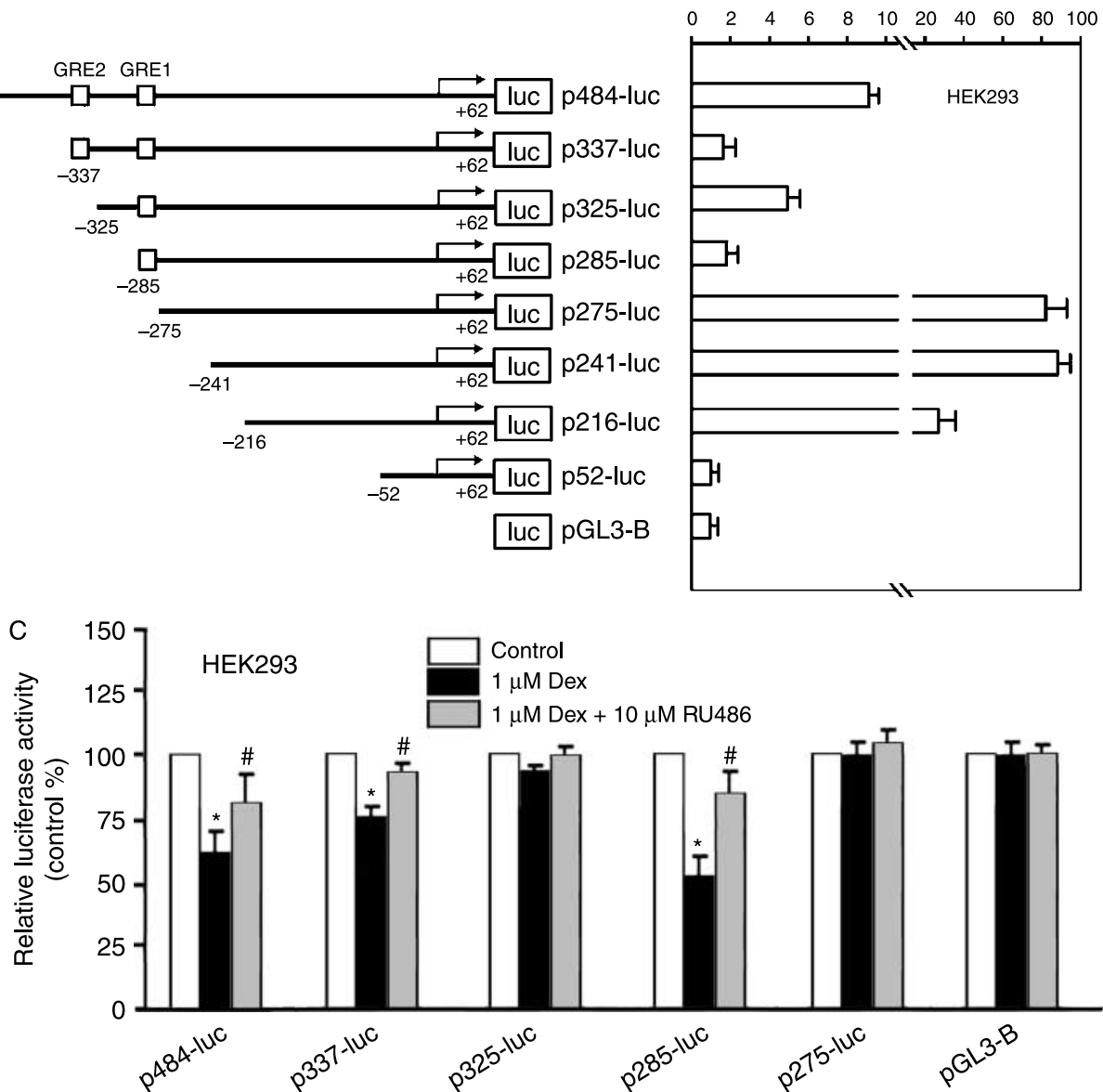

Figure 5 Identification of the cis-elements required for Dex-mediated $h W N K 4$ transcription. (A) Schematic representation of a series of $5^{\prime}$-deleted $h W N K 4$ promoter luciferase constructs. The transcription initiation site is indicated by a bent arrow. Numbers indicate base pairs from transcription start site. (B) The basal activity of the corresponding constructs in HEK293 cells. Luciferase activities were measured by a luminometer (Bethold) normalized to Renilla luciferase activities. Results are expressed as the fold of pGL3-Basic vector that is taken as 1 . The bar is mean \pm S.E.M. from three independent experiments in duplicate for each construct. (C) The comparison of the effects of Dex or Dex with RU486 treatment on the activity of $5^{\prime}$-deleted $h W N K 4$ promoter/luciferase constructs in HEK293 cells. HEK293 cells were transfected with the indicated constructs and treated with (the black bar) or without (the white bar) $1 \mu \mathrm{M}$ Dex or $1 \mu \mathrm{M}$ Dex combined with $10 \mu \mathrm{M}$ RU486 (the grey bar) for $24 \mathrm{~h}$. Luciferase activities were measured by a luminometer (Bethold), normalized to Renilla luciferase activities. Results are expressed as a percentage of the untreated control that is taken as $100 \%$. The bar is mean \pm S.E.M. from three independent experiments in duplicate for each construct $\left({ }^{*} P<0.05\right.$ compared with control; ${ }^{\#} P<0.05$ compared with Dex alone).

\section{Discussion}

Herein, we elucidated for the first time that glucocorticoids suppressed the $h W N K 4$ mRNA expression in HEK293 cells. Furthermore, we analyzed the activity of $h W N K 4$ promoter and identified nGREs in the $5^{\prime}$-flanking region of the $h W N K 4$ gene that functioned in glucocorticoid-mediated repression of the $h W N K 4$ gene expression.

Glucocorticoids are critical physiological regulators of fluid and electrolyte homeostasis. They exert their major function by binding to the intracellular GR that acts as a ligand-activated nuclear transcription factor. Upon ligand binding, the receptor translocates to the nucleus where it interacts with GREs and regulates the transcription of specific gene in target epithelia (Fuller et al. 2000). The effects of glucocorticoids on epithelial transporter genes have been studied in a variety of epithelial cells and tissues (Wald et al. 1998, Snyder 2005). However, to date, there is no data about the modulation of the WNK4 expression by glucocorticoids. We used Dex, a synthetic glucocorticoid that is poor substrate for 11ß-hydroxysteroid 


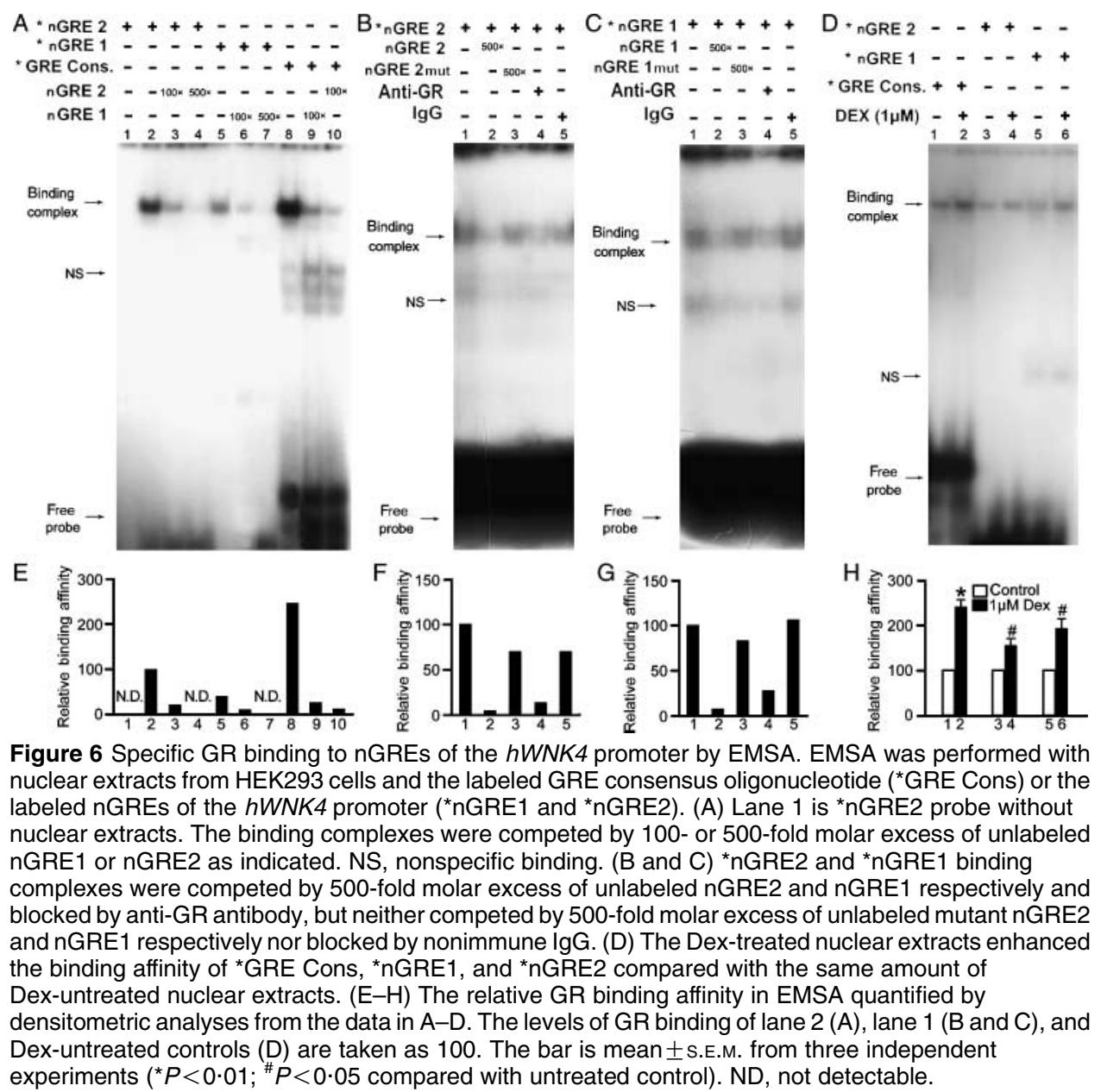

dehydrogenase, to determine the effects of glucocorticoids on the $h W N K 4$ expression in HEK293 cells. Dex decreased $h W N K 4$ mRNA level to $28-35 \%$ of the control after 24-h treatment; and mRNA reduction caused by Dex was effectively prevented by RU486, a synthetic GR antagonist. This long-lasting cellular response to Dex may be caused by transcriptional regulation. Therefore, we identified a transcriptional initiation site of the $h W N K 4$ gene and isolated the $5^{\prime}$-flanking region of the $h W N K 4$ gene to generate the fusion construct with luciferase reporter gene. The luciferase assay demonstrated that Dex inhibited the $h W N K 4$ transcriptional activity in GR-dependent pattern according with the decrease of the $h W N K 4$ mRNA expression.

To our knowledge, GR exists in most cells and acts as ligand-activated nuclear transcription factors to exert the physiological functions of glucocorticoids. Upon ligand binding, the GR translocates to the nucleus where it activates or suppresses gene transcription. In this regard, we further investigated the effect of Dex on the various portions of the $h W N K 4$ promoter in HEK293 cells. We found that the Dex-mediated suppression occurred significantly in p285-luc and p337-luc of the $h W N K 4$ proximal promoter. Moreover, RU486 successfully blocked the reduction caused by Dex treatment. We subsequently identified the negative elements by EMSA and ChIP. The GR binding to negative elements was confirmed by the competition of consensus GRE and interaction with anti-GR antibody. All observations suggested that the negative elements at position -285 and -337 were responsive to glucocorticoids (nGRE1 and nGRE2). Whereas, p325-luc construct containing GRE1 showed no distinct response to Dex in luciferase assay. A possible explanation is that a positive regulatory element harbored in the region -325 to -285 and interfered in its response to Dex. We compared our nGREs with the published nGREs and proved again that nGREs, unlike GREs that share a common consensus, were different in sequence (Malkoski \& Dorin 1999, Radoja et al. 2000, Ou et al. 2001). Considerable evidences indicated that the mechanisms of GR-mediated transcriptional repression involve either GR binding to a simple nGRE such as in the keratin gene (Radoja et al. 2000) or GR interacting with a second 


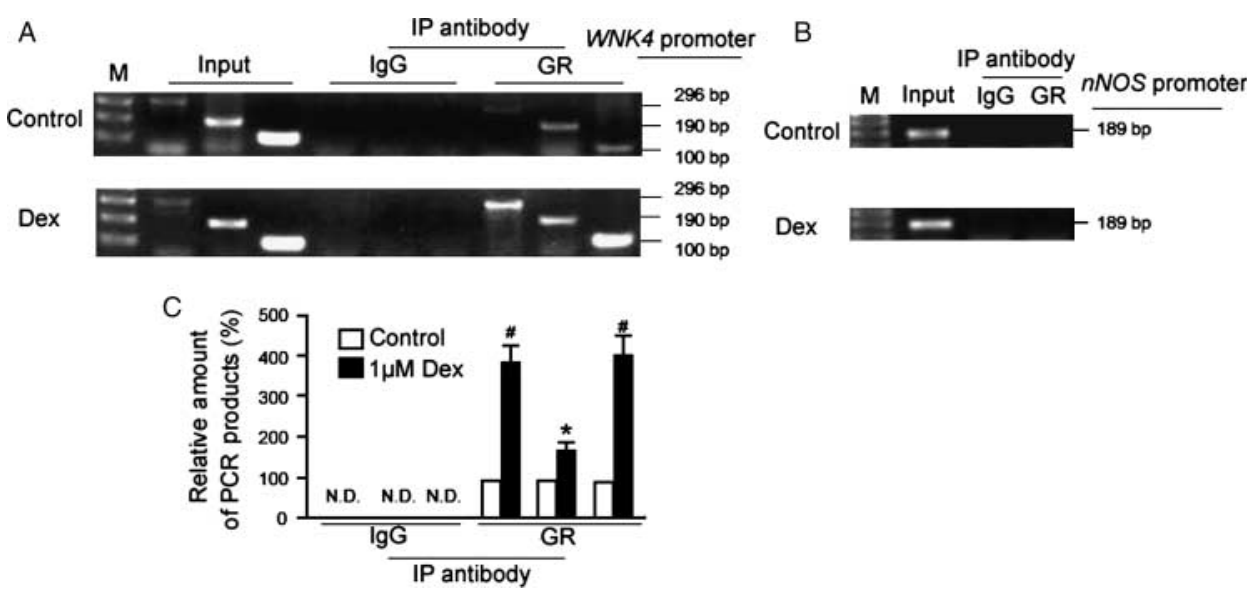

Figure 7 ChIP assays with anti-GR antibody. Chromatin from Dex-treated or -untreated HEK293 cells was immunoprecipitated with anti-GR antibody or nonimmune IgG as a negative control. Input DNA corresponded to the chromatin fragments before immunoprecipitation. (A) The PCR products of $h W N K 4$ promoter were a 296 bp fragment containing both nGRE1 and nGRE2, a 190 and 100 bp fragment containing nGRE2 and nGRE1 respectively. M represented 100 bp DNA ladder. (B) A pair of primers corresponding to $n N O S$ promoter giving a $189 \mathrm{bp}$ fragment was used as a negative control. (C) The relative amount of PCR products representing the GR binding activity in ChIP was quantified by densitometric analyses, normalized to that of input. Results are expressed as a percentage of untreated controls that are taken as $100 \%$ for each group. The bar is mean \pm S.E.M. from three independent experiments ( ${ }^{*}<0.05 ;{ }^{\#} P<0.001$ compared with untreated control). ND, not detectable.

transcription factor, just like that in the osteocalcin, IL-6, and c-fms genes (Meyer et al. 1997, De Bosscher et al. 2000, Flick et al. 2002). Interaction of GR with a second transcription factor is a complex pattern including GR competing an activator binding to GRE (competitive nGRE), GR tethering a factor binding to nGRE (tethering nGRE), and both GR and a factor binding to nGRE (composite nGRE). Our EMSA showed that the in vitro binding was blocked by anti-GR antibody but not by AP-1 (c-jun and c-fos), NF- $\mathrm{BB}$ (p65 and p50), and Oct-1 antibodies (data not shown); ChIP revealed that the binding of GR to the specific nGREs occurred in a native chromosome environment in vitro. However, these data could not rule out other factors interacting with nGREs of $h W N K 4$ promoter in transcriptional repression. Indeed, in the case of competitive nGRE and composite nGRE, GR directly binds nGRE and undoubtedly tethers additional factors. For example, the Dex-mediated repression of the pro-opiomelanocortin $(P O M C)$ gene expression has been initially described as direct binding of GR to a nGRE (Drouin et al. 1993), and subsequent studies have attributed the Dex-mediated repression of the $P O M C$ promoter to tethering the transcription factor Nur77 (Philips et al. 1997). Therefore, we peculated that a combination of these mechanisms could contribute to the glucocorticoid transrepressive effect on the $h W N K 4$ transcription. In addition, the glucocorticoid repression has been reported on posttranscriptional modifications in which the adenylate/uridylate-rich elements (AREs) within the 3'-UTR are now considered central cis-elements in the gene regulation (Stellato 2004). AREs interact with ARE-binding factors and mediate mRNA stability and translation. As we did not find a tandem AUUUA sequence in a TA-rich stretch of the $3^{\prime}$-UTR of the $h W N K 4$ gene, we could not deduce the potential for posttranscription regulation in glucocorticoid repression of the $h W N K 4$ mRNA expression.

In summary, our study demonstrates that glucocorticoids suppress $h W N K 4$ mRNA expression in HEK293 cells, and this suppression can be mediated by two nGREs that are not only required for GR-mediated transcriptional repression but also essential for appropriate transcription level of the $h W N K 4$ gene. Further studies will be necessary to identify the cofactors that participate in the glucocorticoid-mediated repression of the $h W N K 4$ expression. Such analyses of the $h W N K 4$ gene involved in the glucocorticoid regulation will facilitate the identification of responses that are desirable or undesirable for pharmacological effects such as electrolyte disturbance and hypertension. It may be feasible to produce glucocorticoid-related drugs that selectively target the inflammatory process and lead to individualized therapies.

\section{Acknowledgements}

We thank Dr Evans of Howard Hughes Medical Institute for providing pRShGR $\alpha$ vector and Dr Jeunemaitre of 
INSERM U36 College de France for providing $h W N K 4$ cDNA. This work was supported by National Natural Science Foundations of China (30500247, 30300204, and 30370785). The authors declare that there is no conflict of interest that would prejudice the impartiality of this scientific work.

\section{References}

De Bosscher K, Vanden Berghe W, Vermeulen L, Plaisance S, Boone E \& Haegeman G 2000 Glucocorticoids repress NF-кB-driven genes by disturbing the interaction of $\mathrm{p} 65$ with the basal transcription machinery, irrespective of coactivator levels in the cell. PNAS 97 3919-3924.

Buckingham JC 2006 Glucocorticoids: exemplars of multi-tasking. British Journal of Pharmacology 147 S258-S268.

Dickinson H, Walker DW, Wintour EM \& Moritz K 2007 Maternal dexamethasone treatment at midgestation reduces nephron number and alters renal gene expression in the fetal spiny mouse. American Journal of Physiology-Regulatory, Integrative and Comparative Physiology 292 R453-R461.

Drouin J, Sun YL, Chamberland M, Gauthier Y, De Lean A, Nemer M \& Schmidt TJ 1993 Novel glucocorticoid receptor complex with DNA element of the hormone-repressed POMC gene. EMBO Journal 12 $145-156$.

Flick MB, Sapi E \& Kacinski BM 2002 Hormonal regulation of the c-fms proto-oncogene in breast cancer cells is mediated by a composite glucocorticoid response element. Journal of Cellular Biochemistry 8 10-23.

Fuller PJ, Lim-Tio SS \& Brennan FE 2000 Specificity in mineralocorticoid versus glucocorticoid action. Kidney International $\mathbf{5 7}$ 1256-1264.

Kahle KT, Wilson FH, Leng Q, Lalioti MD, O'connell AD, Dong K, Rapson AK, MagGregor GG, Giebisch G, Hebert SC et al. 2003 WNK4 regulates the balance between renal $\mathrm{NaCl}$ reabsorption and $\mathrm{K}+$ secretion. Nature Genetics 35 372-376.

Kahle KT, Macgregor GG, Wilson FH, Van Hoek AN, Brown D, Ardito T, Kashgarian M, Giebisch G, Hebert SC, Boulpaep EL et al. 2004 Paracellular $\mathrm{Cl}$ permeability is regulated by WNK4 kinase: insight in to normal physiology and hypertension. PNAS 101 14877-14882.

Kel AE, Gossling E, Reuter I, Cheremushkin E, Kel-Margoulis OV \& Wingender E 2003 MATCH: A tool for searching transcription factor binding sites in DNA sequences. Nucleic Acids Research 31 3576-3579.

Li Y, Zhao Y, Li G, Wang J, Li T, Li W \& Lu J 2007 Regulation of neuronal nitric oxide synthase exon if gene expression by nuclear factor- $\kappa \mathrm{B}$ acetylation in human neuroblastoma cells. Journal of Neurochemistry 101 1194-1204.
Malkoski SP \& Dorin RI 1999 Composite glucocorticoid regulation at a functionally defined negative glucocorticoid response element of the human corticotropin-releasing hormone gene. Molecular Endocrinology 13 1629-1644.

Meyer T, Gustafsson JA \& Carlstedt DJ 1997 Glucocorticoid-dependent transcriptional repression of the osteocalcin gene by competitive binding at the TATA box. DNA and Cell Biology 16 919-927.

Oakley RH, Webster JC, Jewell CM, Sar M \& Cidlowski JA 1999 Immunocytochemical analysis of the glucocorticoid receptor alpha isoform (GRa) using a GRa-specific antibody. Steroids 64 742-751.

Ortiz LA, Quan A, Zarzar F, Weinberg A \& Baum M 2003 Prenatal dexamethasone programs hypertension and renal injury in the rat. Hypertension 41 328-334.

Ou XM, Storring JM, Kushwaha N \& Albert PR 2001 Heterodimerization of mineralocorticoid and glucocorticoid receptors at a novel negative response element of the 5-HT1A receptor gene. Journal of Biological Chemistry 276 14299-14307.

Philips A, Maira M, Mullick A, Chamberland M, Lesage S, Hugo P \& Drouin J 1997 Antagonism between Nur77 and glucocorticoid receptor for control of transcription. Molecular and Cellular Biology 17 5952-5959.

Radoja N, Komine M, Jho SH, Blumenberg M \& Tomic-Canic M 2000 Novel mechanism of steroid action in skin through glucocorticoid receptor monomers. Molecular and Cellular Biology $204328-4339$.

Snyder PM 2005 Minireview: regulation of epithelial $\mathrm{Na}^{+}$channel trafficking. Endocrinology 146 5079-5085.

Stellato C 2004 Post-transcriptional and nongenomic effects of glucocorticoids. Proceedings of the American Thoracic Society 1 255-263.

Wald H, Garty H, Palmer LG \& Popovtzer MM 1998 Differential regulation of ROMK expression in kidney cortex and medulla by aldosterone and potassium. American Journal of Physiology, Renal Physiology 275 F239-F245.

Wilson FH, Disse-Nicodeme S, Choate KA, Ishikawa K, NelsonWilliams C, Desitter I, Gunel M, Milford DV, Lipkin GW, Achard JM et al. 2001 Human hypertension caused by mutations in WNK kinases. Science 293 1107-1112.

Yamauchi K, Rai T, Kobayashi K, Sohara E, Suzuki T, Itoh T, Suda S, Hayama A, Sasaki S \& Uchida S 2004 Disease-causing mutant WNK4 increases paracellular chloride permeability and phosphorylates claudins. PNAS 101 4690-4694.

Zhao YY, Zhou J, Narayanan CS, Cui Y \& Kumar A 1999 Role of C/A polymorphism at -20 on the expression of human angiotensinogen gene. Hypertension 33 108-115.

Received in final form 12 November 2007

Accepted 14 November 2007

Made available online as an Accepted Preprint

14 November 2007 